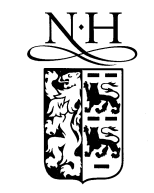

ELSEVIER

\title{
Knowledge reuse in multiple classifier systems ${ }^{1}$
}

\author{
Kurt Dewitt Bollacker * , Joydeep Ghosh \\ Department of Electrical and Computer Engineering, University of Texas, Austin, TX 78712, USA
}

\begin{abstract}
We introduce a framework for the reuse of knowledge from previously trained classifiers to improve performance in a current and possibly related classification task. The approach used is flexible in the type and relevance of reused classifiers and is also scalable. Experiments on public domain data sets demonstrate the usefulness of this approach when one is faced with very few training examples or very noisy training data. (C) 1997 Published by Elsevier Science B.V.
\end{abstract}

Keywords: Knowledge transfer; Multiple classifier; Mutual information

\section{Introduction}

Artificial classifiers depend heavily on the set of training samples to make classification decisions. If the training set insufficiently represents the "essence" of a classification task, then creation of a well generalizing classifier for that task may not be possible. In the construction of artificial classifiers, the inclusion of previously learned knowledge embodied in existing classifiers is a potential approach to the problem of inadequate training data. However, both a suitable representation of the knowledge to be reused, and a mechanism for identification of pertinent knowledge and its incorporation using that representation must be designed. We use attributes of human knowledge reuse as a guide to this design.

One of the most impressive traits of human knowledge reuse is the ability to draw simultane-

\footnotetext{
* Corresponding author.

${ }^{1}$ This work was supported by the Army Research Office contracts DAAH-94-G-0417 and DAAH 049510494 and The National Science Foundation.
}

ously from a large number of previous experiences quickly and easily. Each bit of learned knowledge may not help much, but as a whole, the knowledge gained from experience can paint a very clear picture of the problem domain. Analogously, a practical artificial knowledge reuse system should be able to have good performance scalability with the amount of knowledge reused. Human flexibility in knowledge reuse is also quite notable. Humans can use knowledge learned from a variety of types of experiences without considering how that knowledge was gained. Also, humans are capable of quickly and efficiently picking out learned knowledge that is relevant to the current classification task from their immense body of experience. A flexible knowledge reuse system should be able to take advantage of a diversity of knowledge sources for reuse and have a means to judge the relevance of such knowledge.

\subsection{Previous work}

The most common approach to obtaining a decent generalization given inadequate training sets is to severely constrain the solution space using prior 
domain knowledge. For example, in Bayesian approaches to classification, such knowledge exists in the form of prior distributions assumed for the model parameters and the choice of prior class probabilities. In the machine learning community, the design choice is called "inductive bias" of the classifier. For example, in a decision tree, the bias is indicated by the size of the tree and the variables (or combinations thereof) considered for making the branches. In feed-forward neural networks, the type and number of hidden units, amount and form of regularization (e.g. weight decay) serve to constrain the solution. In all of these approaches, knowledge reuse is indirect. More importantly, they work well only if the inductive bias is a good match to the current problem. This is often difficult to attain in practice.

Some recent work in knowledge reuse has focused on the automated extraction and reuse of knowledge from the data sets of other relevant classifiers, including reuse of the trained classifiers themselves. Under the belief that related classification tasks may benefit from common internal features, Caruana (1995) has created a multilayer perceptron (MLP) based multiple classifier system that is trained simultaneously to perform several related classification tasks. The first layer of the MLP is common to all tasks and the second layer is specific to individual tasks. The first layer is expected to learn common features that are useful to all of the related tasks. Baxter (1994) has developed a rigorous analysis of a similar type of architecture, showing that as the number of simultaneously trained tasks increases, the number of examples needed per task for good generalization decreases. Pratt (1994) has explored a similar knowledge reuse method in which some of the trained weights from one MLP network are used to initialize weights in an MLP to be trained for a later, related task. A different approach is taken by Thrun and O'Sullivan (1996), who proposed a method to estimate classifier relevance by measuring how much better a classifier performs with a reused scaling vector for nearest neighbor classifiers. Tasks with mutually helpful scaling vectors can be "clustered"' into related groups.

Recently, popular approaches such as committees, ensembles, and mixture of experts also use multiple classifiers. However, since all these classifiers try to solve the same task (though they may specialize in different input regions), they are not germane to the work presented here.

\section{Methods}

We describe here an architecture for knowledge reuse from previously trained classifiers. Classifiers trained for the current classification task are called target classifiers while classifiers previously trained to perform other classification tasks are termed support classifiers as indicated in Fig. 1. Our reuse strategy is to apply the input values of each of the training samples available for the target task to all available relevant classifiers. The output class labels of the target and support classifiers are observed by a second stage supra-classifier which makes the ultimate classification $\left(\tilde{c}_{A}(\cdot)\right.$ in Fig. 1$)$. Since no internal information is being used, the support classifiers can be of any type.

\subsection{A few definitions}

Let the target classification task be $A$, and let $A$ have a discrete range $\mathscr{S}_{A}$ and $d$-dimensional input domain space $\mathscr{R}^{d}$. Let $\{x, y\}_{A}: x \in \mathscr{R}^{d}, y \in \mathscr{S}_{A}$ be the set of training examples for task $A$. We assume that $\{x, y\}_{A}$ is sampled from the true distribution for task $A$ with associated random variable $\left(X_{A}, Y_{A}\right) \in$ $\left(\mathscr{R}^{d}, \mathscr{S}_{A}\right)$. Our goal is to find the most likely value of the conditional marginal $Y_{A} \mid\left(X_{A}=x\right)$ and define this maximum likelihood function to be $t_{A}(x)=$ $\operatorname{argmax}_{y} P\left(Y_{A}=y \mid X_{A}=x\right)$. Thus, $t_{A}(\cdot): t_{A}(\cdot) \in \mathscr{S}_{A}$

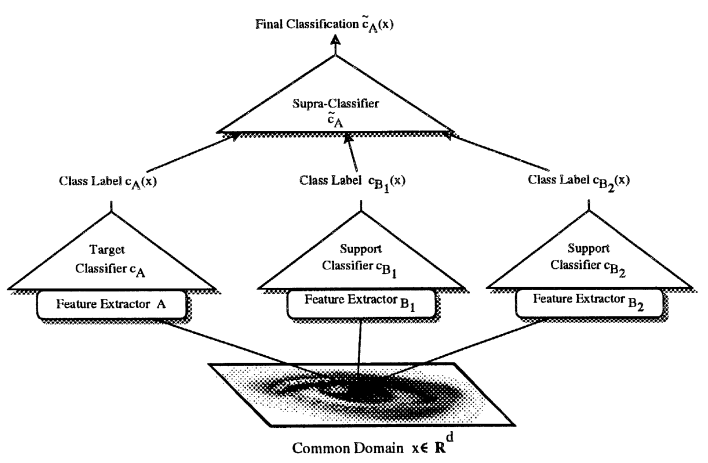

Fig. 1. A supra-classifier reuse architecture. 
is the target function that we would like to approximate using the information in $\{x, y\}_{A}$. Let $c_{A}(\cdot)$ be a function mapping $\mathscr{R}^{d} \mapsto \mathscr{S}_{A}$ which is designed to perform classification task $A$. Let $\mathscr{B}$ be a set of support classification tasks which have the same input domain space $\mathscr{R}^{d}$ as task $A$. Let $\left\{c_{B}(\cdot)\right\}$ : $B \in \mathscr{B}$ be the corresponding set of classifiers where each $c_{B}(\cdot)$ maps $\mathscr{R}^{d} \mapsto \mathscr{S}_{B}: B \in \mathscr{B}$. Let $\hat{X}_{A}$ be the random variable associated with the input values of training sample set $\{x, y\}_{A}$. Let $T_{A}: T_{A}=t_{A}\left(X_{\hat{A}}\right)$ be defined as the random variable associated with the target function of $\hat{X}_{A}$. Similarly, let $C_{A}: C_{A}=c_{A}\left(X_{\hat{A}}\right)$ and $C_{B}: C_{B}=c_{B}\left(X_{\hat{A}}\right)$ be the random variables resulting from the application of $\hat{X}_{A}$ to classifiers $c_{A}(\cdot)$ and $c_{B}(\cdot)$, respectively.

An ideal supra-classifier $c_{A}^{*}(x)$ will always choose the most likely class $y \in \mathscr{S}_{A}$ given the class labels $c_{A}(x)$ and $\left\{c_{B}(x)\right\}: B \in \mathscr{B}$. More specifically, for any given $k_{A}: k_{A} \in \mathscr{S}_{A},\left\{k_{B}: k_{B} \in \mathscr{S}_{B}\right\}: B \in \mathscr{B}$ we can define the maximum probability function $m(\cdot)$ as $m\left(k_{A},\left\{k_{B}\right\}: b \in \mathscr{B}, A, \mathscr{B}\right)=\operatorname{argmax}_{y} P\left(T_{A}\right.$ $\left.=y \mid C_{A}=k_{A},\left\{C_{B}=k_{B}\right\}: B \in \mathscr{B}\right)$. We can then define an ideal classifier based on this maximum probability function as

$c_{A}^{*}(x)=m\left(c_{A}(x),\left\{c_{B}(x)\right\}: B \in \mathscr{B}, A, \mathscr{B}\right)$,

where $c_{A}^{*}(\cdot)$ has an associated random variable $C_{A}^{*}$ : $C_{A}^{*}=c_{A}^{*}\left(\hat{X}_{A}\right)$. In practice, if the number of support classifiers is quite large, Eq. (1) becomes impractical due to the curse of dimensionality (Friedman, 1994). Therefore, we introduce two approximating approaches to Eq. (1) in Section 2.3.

\subsection{Classifier relevance measure}

A measure of relevance of each support classifier to the target classification task would be helpful in the construction of a supra-classifier. We have chosen to use mutual information $I(\cdot ; \cdot)$ ) (a measure of the amount of shared information between two random variables) with the target distribution as a classifier's relevance to that classification task. If $I\left(T_{A} ; C_{B_{1}}\right)>I\left(T_{A} ; C_{B_{2}}\right)$, then we say that $c_{B_{1}}$ " knows", more about $t_{A}(\cdot)$ than does $c_{B_{2}}(\cdot)$. We have empirically demonstrated that mutual information can be used effectively as a relevance measure in our knowledge reuse framework (Bollacker and Ghosh, 1997).

\subsection{Practical supra-classifier methods}

The problem of designing a practical supra-classifier can be thought of as designing a classifier for a task with a large number of discrete features, many or most of which may only be barely useful. We introduce two supra-classifier approaches that are designed to scale linearly in their computational requirements with the number of reused support classifiers in order to satisfy our design goal of scalability.

\subsubsection{Cascaded maximum posterior probabilities}

Let us relabel the support classifiers $\left\{c_{B}(\cdot)\right\}$ : $B \in \mathscr{B}$ in an ordered fashion in the form $\left\{c_{B_{i}}(\cdot)\right\}$ : $i=0 \ldots|\mathscr{B}|-1$ and then revisit Eq. (1) considering only $c_{A}(\cdot)$ and the first support classifier $c_{B_{0}}(\cdot)$ to compute $\hat{c}_{A}^{1}(x): \hat{c}_{A}^{1}(x)=m\left(c_{A}(x), c_{B_{0}}(x), a, B_{0}\right)$. We then progressively update our approximation of Eq. (1) by adding each of the remaining support classifiers in $\mathscr{B}$ one at a time using the form $\hat{c}_{A}^{n+1}(x)=$ $m\left(\hat{c}_{A}^{n}(x), c_{B_{n}}(x), a, B_{n}\right)$. (We define $c_{A}(\cdot)=\hat{c}_{A}^{0}(\cdot)$ for consistency.) As $n$ increases, classification performance on the training example set is strictly non-decreasing (a simple proof omitted for brevity). In a variation to CMAP, we order the cascade of support classifiers by decreasing relevance as a heuristic based on the belief that it would be beneficial to have the most relevant classifiers be earlier in the cascade.

\subsubsection{Hamming nearest neighbor}

If $\delta(\cdot)$ is the indicator function, then the distance measure between two samples $x_{\text {trn }}$ and $x_{\text {tst }}$ can be calculated as $D_{\text {Hamming }}\left(x_{\text {trn }}, x_{\text {tst }}\right)=$ $\Sigma_{B_{i}: i=0 \ldots|\mathscr{B}|-1} \delta\left(c_{B_{i}}\left(x_{\mathrm{trn}}\right) \neq c_{B_{i}}\left(x_{\mathrm{tst}}\right)\right)$. For each test example, the Hamming Nearest Neighbor (HNN) supra-classifier will choose the class label of the training example with the smallest Hamming distance from it. In a weighted variation of this supraclassifier method (WHNN), the distance contribution of each support classifier ( 0 or 1 ) to the total Hamming distance is multiplied by its relevance (mutual information).

\section{Experiments}

If there are too few training examples, or if the examples are too noisy, then good generalization 
may not be possible with the information from the target problem's training examples alone. It is these two cases that we have investigated. In order to test and compare the supra-classifier methods with unaided target classifiers, we took two public domain data sets from the U.C Irvine Machine Learning database and partitioned the examples into two disjoint and unequal sized subsets based on their class labels. The subset with fewer classes became the target task. The other subset was used to create several two-class problems using all combinations of two classes. First, a 20000 example capital English letter data set (LR) donated by David Slate was divided into the target data set consisting of the five classes " $H$ ', " $L$ ', " $O$ ', " $R$ " and ' $S$ ' and 210 support classifiers consisting of two-class classifiers of the other 21 classes. Second, a spoken vowel data set (VOW) contributed by Peter Turney consisted of 990 examples evenly distributed among 11 spoken vowels. The two classes "hud" and "hed" were chosen to form the target classifier task and examples from the remaining 9 classes were used to construct 36 support classifiers.

\subsection{Case of few examples}

The LR data set of 20000 training examples was randomly partitioned into equal sized "base" training and test sets. Both target and support classifier training and test sets were created by taking only examples of the target or support classes respectively from the base training and test sets. The target training set was used to create MLP, single nearest neighbor (1-NN), and C4.5 target classifiers for each target problem. The $210 \mathrm{LR}$ support classifiers were trained MLPs. In order to consider the case of few available target training examples, only a fraction of the available training examples was actually used for training of the target classifier and supra-classifiers. Target training sets of sizes $5,20,40,80,160,320$ and 480 examples were applied to the MLP and 1-NN target classifiers and all of the support classifiers. The outputs of these target and support classifiers were then used as the input vector for each of the supra-classifiers. Average results over 20 trials can be seen in Fig. 2. The WHNN followed by the unweighted HNN supra-classifiers showed better classification performance than the unaided MLP,

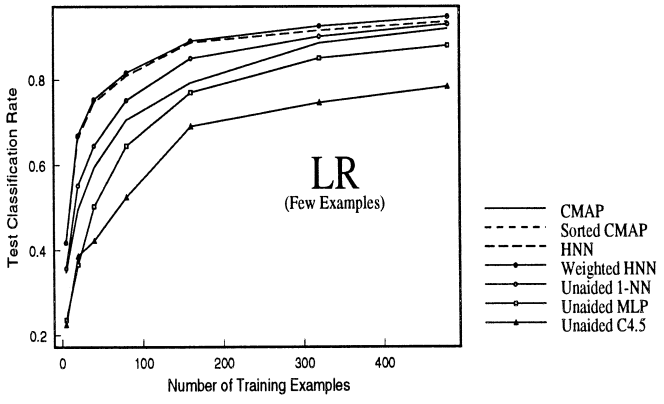

Fig. 2. Classification performance of supra-classifiers and unaided classifiers versus number of training examples on the LR data set.

1-NN and C4.5 classifiers, especially when the number of training examples was very low. The sorted CMAP supra-classifier performed identically to the unaided 1-NN and the unsorted CMAP performed worse. This gives evidence that the information provided by the support classifiers can compensate somewhat for a lack of sufficient training set size.

\subsection{Noisy examples}

A similar experimental setup to the above was made but for the VOW data set in the case of noisy examples. For the target classification problems, Gaussian noise was added to each input vector of the target training set. (The noise covariance matrix was $\sigma^{2} I$.) An MLP target classifier and 36 1-NN support classifiers were used in 100 experimental trials performed over a range $\left(\sigma^{2}=0\right.$ to 16) of noise variances. Average results for the vowel problem are shown in Fig. 3. The performance boost from knowledge reuse in the HNN is quite prominent, but as

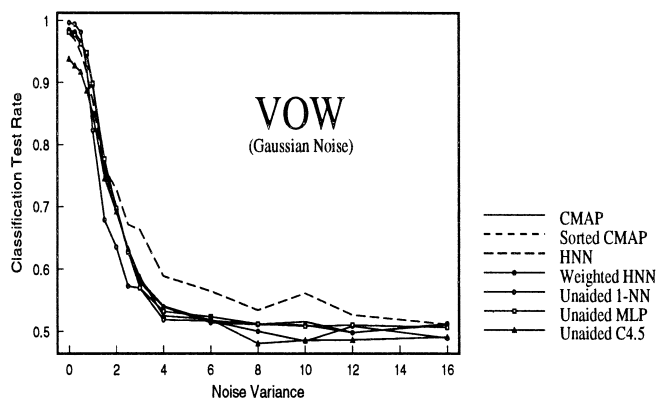

Fig. 3. Classification performance of supra-classifiers and unaided classifiers versus Gaussian noise variance on the vowel data set. 
expected, the advantage disappears as the noise level is lowered.

\section{Conclusions}

In both the case of high noise and of few training examples, knowledge reuse from relevant classifiers via an appropriate supra-classifier improved performance while adhering to the flexibility and scalability design constraints. This happened even though the previously trained support classifiers had no output classes in common with the target classifier. Thus, we have evidence that the knowledge reuse framework presented here can be a practical means for the reuse of knowledge from classifiers that are diverse in form and purpose. We also used a mutual information based relevance measure to guide the construction of some of the supra-classifier methods. This had mixed results for both the CMAP and HNN supra-classifiers, indicating that a relevance measure may help if used carefully.

Although we have shown some encouraging empirical results, there are several directions in which this work can be extended. One of the most important extensions to this work will be application to a truly complex problem domain. We envision the eventual construction of a powerful and broadly applicable "warehouse" of previously constructed reusable classifiers for a large domain of interest (e.g. image databases), where the set of support classifiers will serve as an efficient representation of the problem domain knowledge.

\section{Discussion}

Rhagavan: I was wondering what the relationship is between the classifier that you're trying to design and the classifiers that you use as support?

Bollacker: I am simply using the probabilities, trying to see if there is some correlation. In the letter recognition dataset you have for instance the two classes $\mathrm{H}$ and $\mathrm{O}$. You might expect that a classifier for $\mathrm{N}$ and $\mathrm{Q}$ might be used as a support classifier. You might expect that the support classifier for $\mathrm{N}$ and Q would be able to say something useful about differentiating $\mathrm{H}$ and $\mathrm{O}$, since the features for that dataset were statistics on the shapes of the letters and since $\mathrm{N}$ is similar to $\mathrm{H}$ and $\mathrm{Q}$ is similar in shape to
O. So, you would hope that there would be some useful information that you could derive from that $\mathrm{N}$, Q classifier.

Van Dyck: I want to point out an analogy between what you did and the infotree method which I presented. There we considered the pixel as the most primitive classifier. We combined pixels using mutual entropy, to get a higher level classifier. This is repeated in a kind of tree fashion until the final classifier gives the whole pattern.

Bollacker: When I first started looking at this, I thought of something like that. The first domain I considered was images. But I decided that there was too much overhead building a set of classifiers for an image domain. So we used these simpler datasets. In the long term, towards building a "warehouse" of classifiers, you would start to build classifiers that say very simple things about the image domain. Then using those, you might be able to build classifiers that say slightly more complex things, and so on, in some sort of hierarchy like you presented. But that seems to be a much longer-term project than what we have done so far.

Loew: When you showed the curves of performance, it would have been helpful if we would have been able to see some error bars on those points, so we could have a feel for whether the differences between them were significant or not. But perhaps more importantly, I am wondering about your nearest neighbour classifier, which seems to be almost the best or second in the two cases. I wonder whether, if you had gone to some $K$-nearest neighbour classifier, if the performance would have come very close. Do you have any feel for that?

Bollacker: About the error bars: the reason that I did not put these on is that the graphs are already relatively cluttered. So I just made sure that I performed a large enough number of trials to make sure that the error bars would be small. Regarding the other question: are you talking about a single nearest neighbour supra-classifier?

Loew: No, I think at the lower level.

Bollacker: You are talking about the unaided single nearest neighbour classifier. Actually, I gave these 
unaided classifiers the benefit of the doubt. And it turned out in all the data sets that I used, that for all $K$-nearest neighbour classifiers, the single nearest neighbour classifier worked better than those with larger $K$.

Loew: Do you have any feel for why that was so?

Bollacker: Well, in fact that was not true in all the data sets that I used, but it was true for these two. So I just decided that I would choose the best one and use that for comparison.

Roli: I would like to have a clarification. Is it correct to say that the concept of knowledge reuse can be regarded as a problem of identifying in a library of classification algorithms, the most independent ones, that is the algorithms that make uncorrelated errors? Because, of course, classifiers that make uncorrelated errors are the classifiers most promising to be combined. In your opinion, is this true?

Bollacker: We haven't done an analysis to look at error correlation. And certainly what you are sug- gesting might result in a better relevance measure. So that is certainly something to look at. It could be added to the list of future things to do.

\section{References}

Baxter, J., 1994. Learning internal representations. Ph.D. Thesis, The Flinders University of South Australia.

Bollacker, K.D., Ghosh, J., 1997. A scalable method for classifier knowledge reuse. In: Proc. 1997 Internat. Conf. on Neural Networks.

Caruana, R., 1995. Learning many related tasks at the same time with backpropagation. Adv. Neural Inform. Process. Systems 7, 657-664.

Friedman, J.H., 1994. An overview of predictive learning and function approximation. In: Cherkassky, V., Friedman, J.H., Wechsler, H. (Eds.), From Statistics to Neural Networks, Proc. NATO/ASI Workshop. Springer, Berlin, pp. 1-61.

Pratt, L.Y., 1994. Experiments on the transfer of knowledge between neural networks. In: Hanson, S., Drastal, G., Rivest, R. (Eds.), Computational Learning Theory and Natural Learning Systems, Constraints and Prospects, Chapter 19. MIT Press, pp. 523-560.

Thrun, S., O’Sullivan, J., 1996. Discovering structure in multiple learning tasks: The TC algorithm. In: Proc. 13th Internat. Conf. on Machine Learning. 